\title{
The New Coronavirus (COVID-19) Declared as a Global Pandemic
}

\section{Moure UAE, Koundi LM, Lambert N, Nzaou SAE and Xie J ${ }^{*}$}

Institute of Modern Biopharmaceuticals, State Key Laboratory Breeding Base of Eco-Environment and Bio-Resource of the Three Gorges Area, Key Laboratory of Eco-environments in Three Gorges Reservoir Region, Ministry of Education, School of Life Sciences, Southwest University, Beibei, Chongqing, China

${ }^{*}$ Corresponding author: Xie J, Institute of Modern Biopharmaceuticals, State Key Laboratory Breeding Base of Eco-Environment and Bio-Resource of the Three Gorges Area, Key Laboratory of Eco-environments in Three Gorges Reservoir Region, Ministry of Education, School of Life Sciences, Southwest University, Beibei, Chongqing, China, E-mail: georgex@swu.edu.cn

Citation: Moure UAE, Koundi LM, Lambert N, Nzaou SAE, Xie J (2020) The New Coronavirus (COVID-19) Declared as a Global Pandemic. J Respir Dis Treat 1(1): 106. doi: 10.15744/2767-4649.1.106

Received Date: March 13, 2020 Accepted Date: April 15, 2020 Published Date: April 17, 2020

\begin{abstract}
The lately declared-coronavirus (COVID-19) is being the main threat worldwide due to its high incidence, morbidity and global economic impacts. It is easy to spread from person to person, through infected surfaces, and the absence of vaccine make the situation more difficult to handle. This review aims to alert the pandemic evolution taken by the actual outbreak of the COVID-19, and therefore calls the highest institutions of the global health namely the World Health Organization (WHO) to reinforce the measures taken in the fight against this new virus by working tightly with each affected region and the emergency in elaborating a new vaccine.

Keywords: COVID-19; Fast evolution; Pandemic

List of abbreviations: COVID-19: New Wuhan Coronavirus 2019; MERS: Middle East Respiratory Syndrome; SARS: Severe Acute Respiratory Syndrome; USA: United States of America; WHO: World Health Organization; (H1N1) pdm09: The 2009 H1N1 Pandemic Virus
\end{abstract}

\section{Introduction}

The COVID-19 is a new respiratory virus of the coronavirus family discovered in December 2019 in the Wuhan city in China [1]. It is supported that this new virus comes from an animal (bat, pangolin, etc.) and has jumped to humans through direct contacts as the outbreak started in Wuhan's Huanan Seafood Wholesale Market [2]. The transmission is made through contact from an infected person to another person via the swallowing of aerosol duplets or through contacts with infected surfaces or objects [3]. This new virus takes almost two weeks of incubation before the apparition of the first symptoms, which are fever, cough and trouble breathing. In more complicate cases, it can cause severe respiratory illness similar to SARS and MERS, and lead to death [3]. In the urge of the situation, many assays have come to demonstrate the apparent efficacy of chloroquine on the COVID-19 [4-6]. Until now, no vaccine has not been elaborated yet. Antibiotics cannot be used in the treatment, as the COVID-19 is a virus. The use of antivirals is experimentally considered but not approved to treat the COVID-19 [7]. The treatment used is supportive (supplemental oxygen, mechanical ventilation) and just alleviates the pains of patients. Infection prevention and control are highly recommended [8].

Days after the outbreak in Wuhan, some cases have been detected out of the epicenter and abroad like Japan, France and the USA [9], etc. After investigations, most of cases reported abroad had travel history related to China in the two past weeks, and the remaining cases were due to secondary transmission [10]. In China, during the period of mid-January to early February, the peak of spreading of the COVID-19 was increasing, causing thousands of infected and hundreds of deaths [11,12]. The WHO declared the actual outbreak as a Public Health Emergency [13], giving some measures (regular hands washing, wearing of the mask when going outside, avoiding crowded places) which have led some countries to take immediate precautions (flights stopping, borders closing, fourteen days of quarantine when coming from high risk areas, etc.) to handle the epidemic situation.

This is not the first time for the world to face a global health crisis. The 1918 influenza pandemic (H1N1) with an unknown origin, was the deadliest pandemic in recent history, with about 500 million (1/3 of the world's population) cases, associated with at least 50 million deaths worldwide [14,15]. In Southern China (Guangdong), an outbreak of SARS-CoV raised in 2003. About 8098 cases of infections related to SARS were reported globally, associated with 774 deaths $[15,16]$. In 2009, the (H1N1) pdm09 virus caused 
a pandemic andkilled about 151 700-575 400 people worldwide, with 60.8 million cases and 12469 deaths in the United States [17]. From 2014-2016, the Ebola outbreak came out. It was very deadly but less contagious than the COVID-19, as it spread most of the cases through bodily fluids like sweat and blood during the last stages of the disease. Globally, 28652 cases were reported across 10 countries associated with 11325 deaths [15]. All those previous outbreaks should have taught the world and challenged it to invest more in the health system and scientific research. Therefore and all means used for preparedness and response to any unexpected eruption.

What could be the reasons or factors that led this health crisis to spread all over the world, to cause such deaths and reach a pandemic level? Here, by raising the alarming and dramatic level reached by the new coronavirus, we aim to emphasize the eventual reasons or factors that might have contributed to this global health crisis and urge for immediate actions to control the spread of the disease and the need to elaborate a vaccine soon.

\section{Results}

\section{WHO areas with reported confirmed cases of COVID-19}

By 11 March 2020, all the regions worldwide defined by the WHO have reported at least one case of the COVID-19 (Figure 1). Among them are the Western Pacific Region, the European Region, the South-East Asia Region, the Eastern Mediterranean Region, and the Region of the Americas and the African Region.

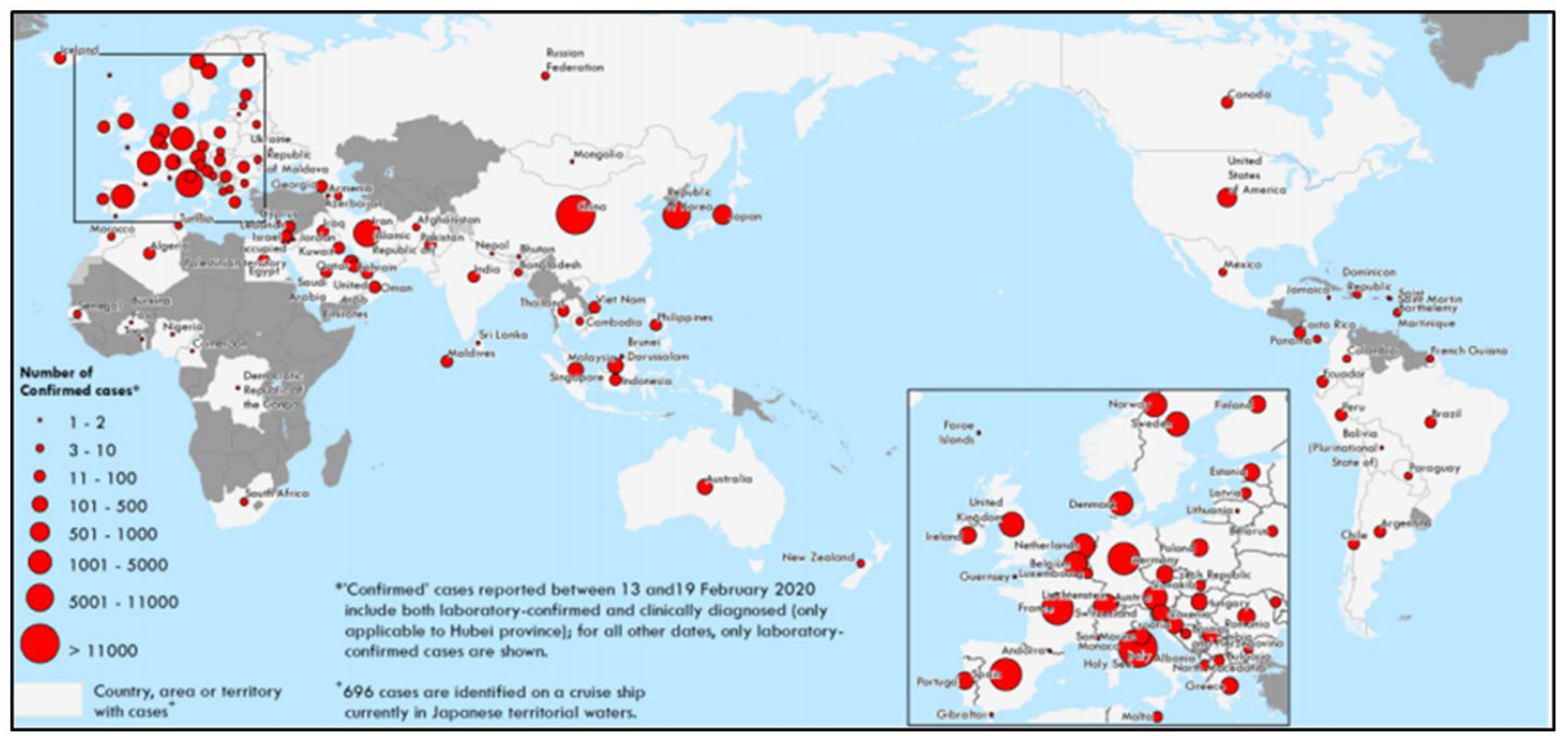

Figure 1: Countries, territories or areas with reported confirmed cases of COVID-19, by 11 March 2020 (Taken from coronavirus disease 2019 (COVID-19), Situation Report -51, WHO)

\section{Global transmission classification of the COVID-19}

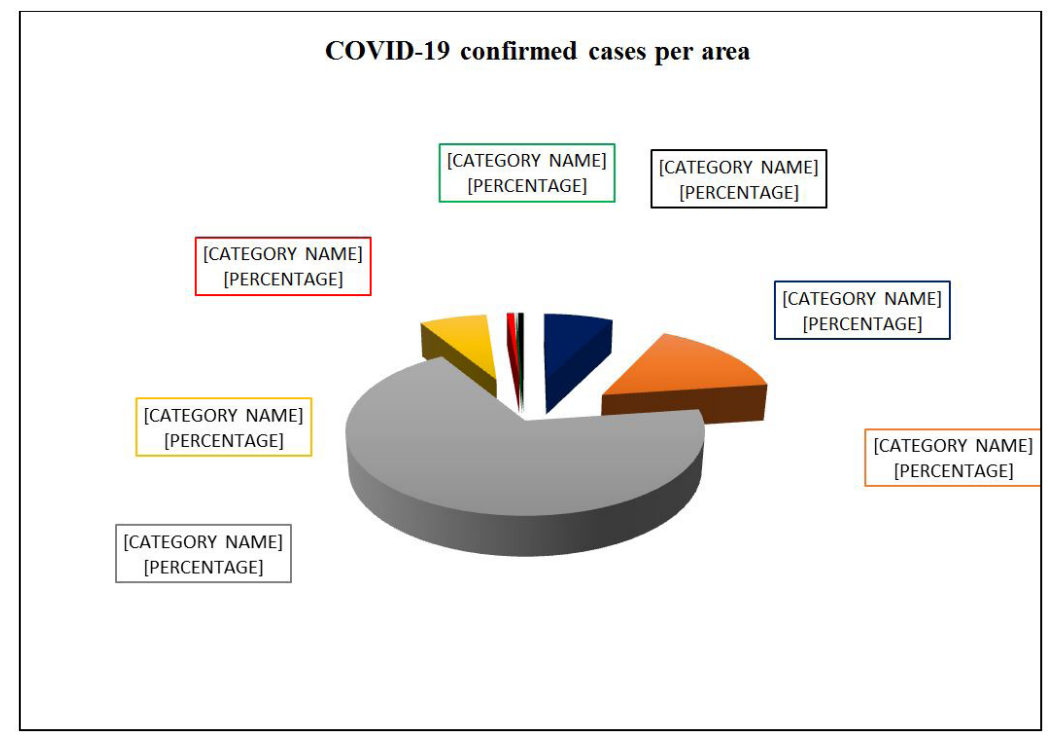

Figure 2: Percentages of COVID-19 cases per area, by 11 March 2020

(China has been included in South-East Asia area) 


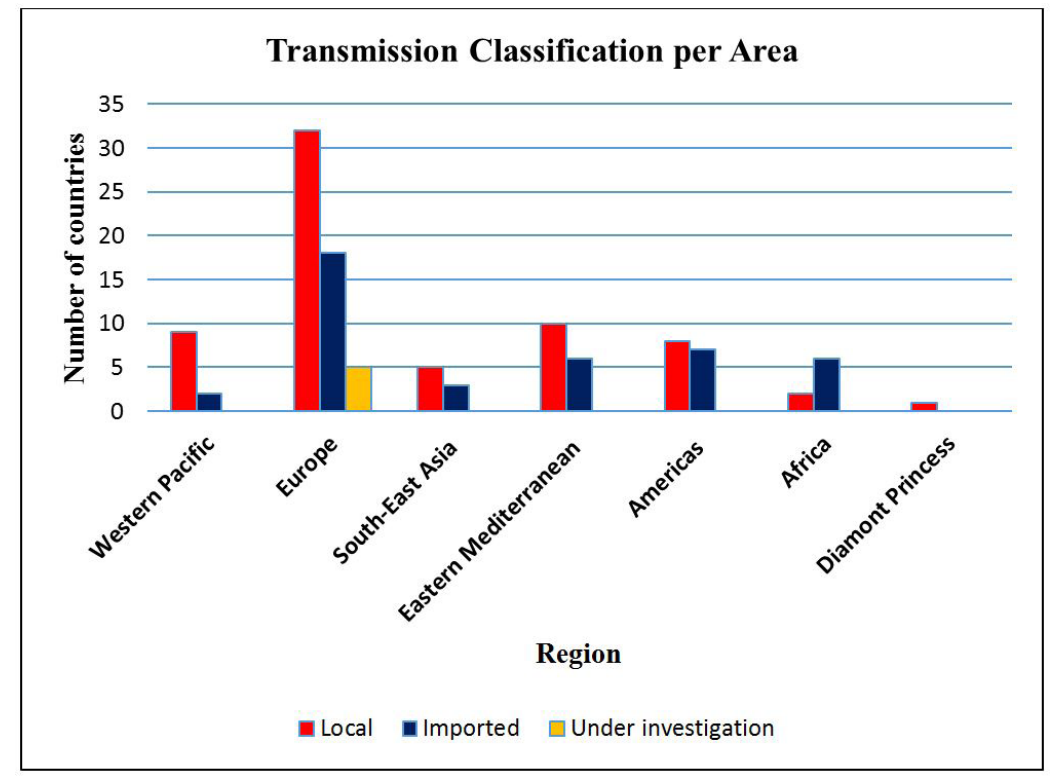

Figure 3: Transmission classification found in different areas and the number of countries per area is depicted (By 11 March 2020)

Two types of transmission have been noticed, the imported transmission and the local or community transmission. However, some cases are still under investigation. According to the Figure 2, the European Region is the region with the highest number of countries with imported cases, followed by the Americas, Africa and Eastern Mediterranean regions, while South East Asia and the Western Pacific have fewer countries with this type of transmission of the COVID-19 by 11 March 2020. The European Region contains most countries with local transmission of the COVID-19, seconded by the Eastern Mediterranean, the Western Pacific Region, the Americas and South East Asia Regions (Figure 3). Africa is the region with fewest countries with important cases.

\section{COVID-19 confirmed cases and deaths over the world}

The Table 1 tells about the outbreak in China. A total of 80955 confirmed cases have been confirmed with 3162 deaths. Out of China, 37364 cases of COVID-19 have been reported in 113 countries and territories with 1130 deaths over the world (Table 2). Globally, 118319 (in and out of China) confirmed cases and 4292 deaths have been recorded by 11 March 2020. Per region, the percentage of COVID-19 cases is as the following: the South East Asia Region (69\%), the European Region (15\%), the Eastern Mediterranean and the Western Pacific Regions (7\%) respectively are the more prevalent. The Regions of the Americas and Africa are the regions with the lowest number of COVID-19 cases.

\begin{tabular}{|c|c|c|}
\hline Location (China) & Cases & Deaths \\
\hline Hubei province (Wuhan) & $67773(83.72)$ & $3046(96.33)$ \\
\hline Others parts of China* & $13182(16.28)$ & $116(3.67)$ \\
\hline Total & $80955(100)$ & $3162(100)$ \\
\hline
\end{tabular}

\begin{tabular}{|c|c|c|c|}
\hline Area (out of China) & NC & Total confirmed cases & Total death \\
\hline Western Pacific & 11 & $8824(23.61)$ & $76(6.72)$ \\
\hline Europe & $48^{*}$ & $18140(48.54)$ & $717(63.45)$ \\
\hline South-East Asia & 8 & $160(0.42)$ & $2(0.17)$ \\
\hline Eastern Mediterranean & $16^{\#}$ & $8578(22.95)$ & $300(26.54)$ \\
\hline Americas & 15 & $927(2.48)$ & $28(2.47)$ \\
\hline Africa & 8 & $39(0.10)$ & $0(0)$ \\
\hline Diamond Princess & 0 & $696(1.90)$ & $\mathbf{1 1 3 0}(\mathbf{1 0 0})$ \\
\hline Total & $\mathbf{1 0 6}$ & $\mathbf{3 7 3 6 4}$ &
\end{tabular}

*The total number of cases and deaths of French Guiana, Martinique, Saint Martin, Saint Barthelemy; Faroe Islands, Gibraltar, Guernsey territories have been considered together with total cases and deaths of the European region. "Data of the Occupied Palestinian territory has been included in the Eastern Mediterranean region data. In brackets: the percentage.

Table 2: Confirmed cases and deaths caused by the COVID-19 infection out of China, 11 March 2020 


\section{Discussion}

To date, every parts of the whole world have reported a least one case of the COVID-19 in a very short time. A pandemic sparked by a coronavirus has never been before seen. This is the first pandemic caused by a coronavirus [18]. By 11March 2020, the COVID-19 has caused more than 4292 deaths and 118319 infected globally, according to the WHO report. Despite the high morbidity (80 955 infected) and mortality (3 162 deaths) due to the high peak of the outbreak in the past weeks, the number of new cases and deaths are falling down in China (31 new infected and 22 new deaths) [13]. In opposition, the other parts, without any exception are now struggling with this threat. Out of China, 37367 confirmed cases and 1130 deaths have been notified in 113 countries, territories or areas. The global incidence of the COVID-19 is increasing, with 4592 new cases and 258 new deaths [13]. The WHO has finally declared the new coronavirus as a pandemic in observation of the alarming levels of spread and severity, the number of countries affected, and the number of people passed away [19].

The world has known many outbreaks before in the past with their social impacts. The Spanish flu in 1918, the SARS from 2002 to 2004, the Influenza (H1N1) pandemic in 2009 and the Ebola outbreak in 2014-2016 [20]. However, many factors might explain the quick pandemic trend took by this health crisis:

First, the globalization, the advancements in international transportation systems and excessive globalization may have made it easy for COVID-19 to spread from country to country and from a continent to another one.

Second, the transmission route. COVID-19 is very contagious. Airborne transmitted-viruses are easy to spread from one person to another one. Crowed places and cities with high populations like Wuhan (8 364977 people) [21], the epicenter of the outbreak are suitable for the fast spread of such a virus. The quarantine of the city of Wuhan may have largely contributed to decreasing the spread of the disease out of the latter. In addition, unlike the SARS, which had not the fitness to persist in the human population, the COVID-19 is able to spread and thrive in the human body. In addition, because the COVID-19 is a new virus, people do not have the herd immunity to this pathogen to reduce its transmission like the seasonal flu. This might allow the high spread and the fatal effect of the COIVD-19.

Third, the incubation period the COVID-19, which lasts about fourteen days is longer than the seasonal flu. During this period, people are asymptomatic and can spread the virus easily to other [22]. This makes it difficult to identify and to contain. With the H1N1 flu in 2009 and the Ebola outbreak in 2014, children were the most affected group [20]. In contrast, with the COVID-19, old adults seem to be the target group as they have most of the underlying illnesses (cardiovascular diseases, diabetes, etc.). Children who are spared and experiencing mild symptoms contaminate the latter.

Finally, the late consideration of the COVID-19 disease as a global health threat might also have played a crucial role in the fast spreading of the infection worldwide at a pandemic level. In addition, this also had an impact on the late developments of a vaccine by the laboratories, the engagement of the whole world to join as one to fight against this pandemic by finding solutions.

\section{Conclusion}

In short, given the stage reached by this health crisis, an urgent response is required in order to avoid exorbitant losses. The WHO should strengthen previously taken measures, find new solutions to quickly control and contain the spread of the COVID-19, in partnership with all regions. Joint efforts must be made and the need for a vaccine is required since there is no specific treatment. However, a deep consideration of the factors that might lead this ongoing outbreak to quickly reach the pandemic level in short times is required and will be of a great interest in the preparedness and response of the world to any new virus invasion.

\section{Acknowledgment}

The authors are grateful to Professor Jianping Xie for his ideas and critical reading of the manuscript.

\section{Author contributions}

The authors have contributed equally to this work.

\section{Conflict of interest}

The authors have no conflict of interest to declare.

\section{References}

1. Wang C, Horby PW, Hayden FG, Gao GF (2020) A novel coronavirus outbreak of global health concern. Lancet 395: 470-3.

2. Lu H, Stratton CW, Tang Y-W (2020) Outbreak of Pneumonia of Unknown Etiology in Wuhan, China: The Mystery and the Miracle. J Med Virol 92: 401-2.

3. Centers for Disease Control and Prevention (2020) How COVID-19 Spreads, USA.

4. Wang M, Cao R, Zhang L, Yang X, Liu J, et al. (2020) Remdesivir and chloroquine effectively inhibit the recently emerged novel coronavirus (2019-nCoV) in vitro. Cell Res 30: 269-71. 
5. Gao J, Tian Z, Yang X (2020) Breakthrough: Chloroquine phosphate has shown apparent efficacy in treatment of COVID-19 associated pneumonia in clinical studies. Biosci Trends 14: 72-3.

6. The National Health Commission of the People's Republic of China (2020) Audio transcript of the news briefing held by the State Council of China on February 17, 2020, China.

7. NSW Health (2020) NSW Health Interim Guidance on use of antiviral therapy in COVID-19, China.

8. Centers for Disease Control and Preventio (2020) Information for Clinicians on Therapeutic Options for Patients with COVID-19, USA.

9. Centers for Disease Control and Prevention (2020) Confirmed 2019-nCoV cases globally, USA.

10. World Health Organization (WHO) Novel Coronavirus (2019-nCoV), Situation Report-12, Switzerland.

11. Liu Y, Gayle AA, Wilder-Smith A, Rocklöv J (2020) The reproductive number of COVID-19 is higher compared to SARS coronavirus. J Travel Med 27: 10.1093/ $\mathrm{jtm} / \mathrm{taaa} 021$.

12. National Health Commission China (2020) Update on pneumonia of new coronavirus infection, China.

13. World Health Organization (WHO) Novel Coronavirus (2019-nCoV), Situation Report-51, Switzerland.

14. Centers for Disease Control and Prevention (2020) 1918 Pandemic (H1N1 virus), USA.

15. Healthline (2020) Here's How COVID-19 Compares to Past Outbreaks, USA.

16. Ahmed SF, Quadeer AA, McKay MR (2020) Preliminary Identification of Potential Vaccine Targets for the COVID-19 Coronavirus (SARS-CoV-2) Based on SARS-CoV Immunological Studies. Viruses 12: 10.3390/v12030254.

17. Centers for Disease Control and Prevention (2020) 2009 H1N1 Pandemic (H1N1pdm09 virus), USA.

18. World Health Organization (WHO) WHO Director-General's opening remarks at the media briefing on COVID-19, 11 March 2020, Switzerland.

19. World Health Organization (WHO) World-Health-Organization Statement on the second meeting of the International Health Regulations (2005) Emergency Committee regarding the outbreak of novel coronavirus (2019-nCoV), Switzerland.

20. Healthline (2020) How deadly is the coronavirus compared to past outbreak, USA.

21. World Urbanization Prospects (2020) United Nations population estimates and projections of major Urban Agglomerations, USA.

22. Centers for Disease Control and Prevention (2020) Coronavirus Disease 2019 (COVID-19) Symptoms \& Testing, USA.

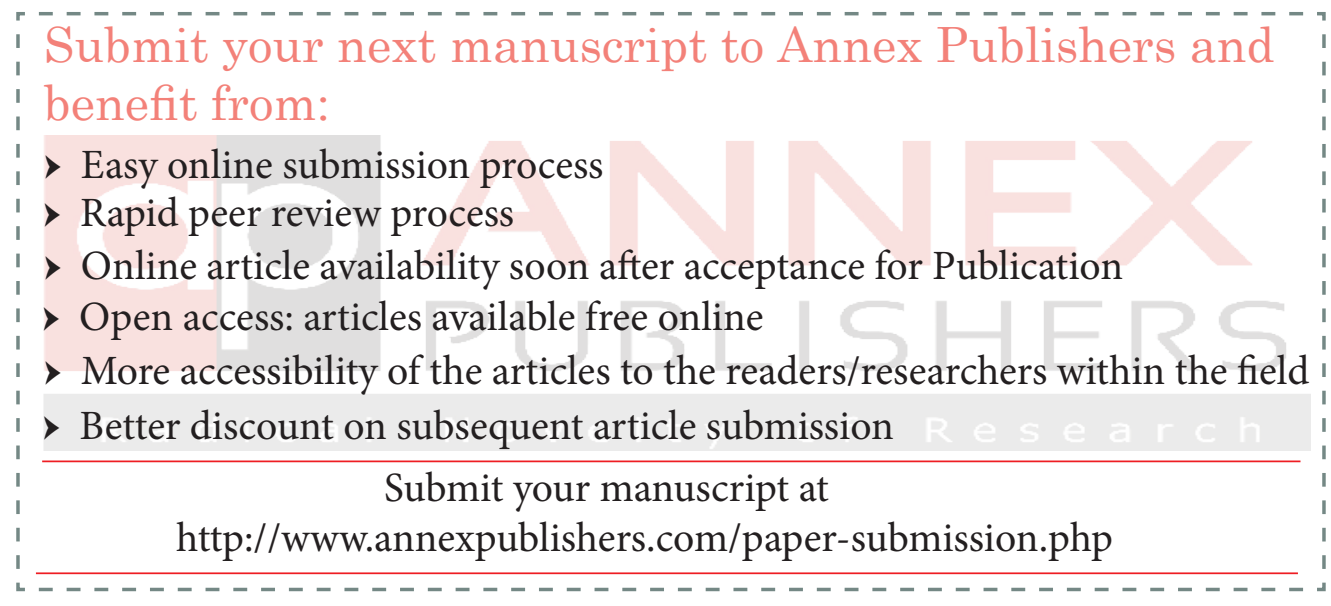

